\title{
Value of a Friend, a Friend of Your Friend, and a Friend of the Friend of Your Friend
}

\author{
Kuninori Nakamura \\ Seijo University, Tokyo, Japan
}

\begin{abstract}
Jones and Rachlin (2006) found that the amount of money a person is willing to forgo in order to give $\$ 75$ to another person decreased as a hyperbolic function of perceived social distance, in the same way as occurs in intertemporal choice. This study aimed to extend this finding to the domain of social networks, in which social distance is defined by degrees of separation. A total of 334 participants responded to tasks very similar to those in Jones and Rachlin (2006), except that they were required to choose whether they would prefer to receive an amount of money for themselves only or an amount of money for themselves and a person who is $n$ degrees of separation from them up to six degrees. The results show that the hyperbolic function fit the data well, and that several processes appear to contribute to the judgments made in the experimental tasks.
\end{abstract}

Keywords: social discounting, $n$ degrees of separation, intertemporal discounting, probability discounting

\section{Social Discounting in $\boldsymbol{n}$ Degrees of Separations}

In modern society, people must use money to live. However, as a matter of course, people cannot use all their money at once. Rather, people must allocate it between several purposes or several time periods. They distribute money between several stock options so as not to lose all their money at once. They save money for the future to avoid financial difficulties when they are old. Additionally, we also must share money with others in order to prevent poverty. As these examples show, how people use money is of vital importance in life, and appears to be organized according to several dimensions.

According to Simon (1995), a person's allocation of available goods can be described in terms of a three-coordinate system: One is the coordinate of their own current consumption, included in which is the concept that a person has several selves corresponding to their various positions, such as the family self or working self, and so need to allocate goods to these selves. The second is a coordinate of later times, representing sequential temporal persons as different from each other. The third is consumption by other people, according to which the feeling of sympathy between people may be measured by a discount factor. Simon (1995) suggested that this discount function may be similar to that of intertemporal discounting.

Consumption in later time periods corresponds to what is called intertemporal discounting. Many studies have demonstrated that people discount the value of goods as time goes by. Generally, it is known that people prefer small but immediate goods to large but delayed goods (e.g., Lowenstein \& Prelec, 1998). To account for this preference, exponential and hyperbolic functions have been proposed.

An exponential discounting function has a form in which the discounted value $v$ of rewards $V$ is expressed

Kuninori Nakamura, associate professor, Faculty of Social Innovation, Seijo University. 
as follows:

$$
v=V e^{-k D}
$$

where $v$ and $V$ are the discounted and undiscounted reward values, respectively, and $D$ is the time delay.

Alternatives to exponential discounting have been proposed by psychologists, behavioral ecologists, and behavioral economists. One major alternative proposal is that the discounting function is hyperbolic (e.g., Mazur, 1987):

$$
v=\frac{V}{1+k D}
$$

Until now, many studies have demonstrated that the hyperbolic function, rather than the exponential function, is the most appropriate because it fits the data better than the exponential function, under various experimental conditions (for a review, see Green \& Myerson, 2002).

On the basis of findings within the intertemporal choice literature, Jones and Rachlin (2006) investigated whether discounting similar to intertemporal choice could also be found in Simon's third coordinates. In their experiments, Jones and Rachlin (2006) required participants to imagine that they had made a list of the 100 people closest to them in the world, ranging from their dearest friend at position \#1 to a mere acquaintance at $\# 100$, and then participants answered whether they would forgo a fixed amount of money to give it to another person or not. The results showed that the amount of money people were willing to give to another person decreased as a hyperbolic function of the perceived social distance between them, indicating that the discounting function with regard to social distance is similar to that in intertemporal choice, as Simon (1995) claimed. This study aims to extend the findings of Jones and Rachlin (2006) by considering another type of social distance.

Recent studies in network science have begun to pay attention to the concept of human society considered as a network (Barabási, 2002; Christarski \& Fowler, 2009; Milgram, 1967). You have a friend, and your friend has a friend. If you do not know the friend of your friend, you can still have a link to the person via your friend. You can extend such connections infinitely. In this vein, our society is a network of friend connections, and much research has paid attention to various aspects of our society considered as such a network (Barabási, 2002; Christarski \& Fowler, 2009).

In a social network, the $n$th degree of separation can be considered as one type of social distance between two persons. The degree of separation refers to the number of links via friends between two persons. For example, your friend is a friend of the first order, because there is one link between you and your friends, and a friend of your friend is of the second order because there are two links; one is between you and your friend, and the other is between your friend and the friend of your friend. The number of links $(n)$ between two persons can be increased infinitely, and as $n$ increases, the social distance from you increases. For example, a second order friend is more remote than a first order friend, because you have not met the former whereas you directly know the latter. In addition, a third order friend is more remote than a second order friend, because even your friend does not know the former person. If the degree of separation is taken as social distance, the following questions arise: Does social discounting also appear with this type of social coordinate? If so, is it hyperbolic or exponential? The first purpose of this study is to address these questions.

A second purpose of this study is to explore the relationship between social ranking and degrees of separation as measures of social distance. Although both social ranking and degrees of separation can be 
considered as indices of social distance between persons, their meanings are a little different. Whereas the former measure reflects the distance among in-group members because it assumes that the ranking orders the 100 people closest to a person, from the dearest friend to a mere acquaintance, the latter measure contains not only in-group but also out-group individuals, because one cannot know a friend whose degree is of more than two. Thus, even if hyperbolic discounting is found in the nth degree of separation measure, the relationship between social ranking and degrees of separation would still be an interesting question. Therefore, this study also aims to address this issue.

For this research two empirical studies have been performed. Study 1 was to examine whether social discounting occurs with social distance in terms of degrees of separation. Study 2 explored the relationship between degrees of separation and closeness ranking, as used in Jones and Rachlin (2006).

\section{Study 1}

Study 1 required participants to answer whether they would prefer (a) an amount of money for themselves or (b) an amount of money for themselves and the person who is $n$ degrees of separation from them, up to 6 degrees of separation. By this procedure, we tried to determine the amount of money forgone to give a person 60,000 yen. This procedure is almost the same as that of Jones and Rachlin (2006), except that the closeness ranking had been exchanged for degrees of separation.

\section{Procedure}

One hundred and nineteen undergraduates participated in Study 1 for course credits, and all materials and response formats were provided in booklets. The booklets explained the meaning of the degrees of separation on the first page, and the experimental tasks began on the second page. The second page contained the following instructions:

You know a person who is called "your friend". The minimal condition for a person to be called a friend is that you and s/he know each other. In addition, there is a "friend of your friend" who is known to your friend but unknown to you. This "friend of your friend" can also know a "friend of the friend of your friend" and a chain of friends can extend infinitely. Thus, we connect to various people through friends, friends of friends, and friends of friends of friends, and so on, although most of them are unknown to you.

Next, you will be asked to make a series of judgments based on your preferences. On each line, you will be asked if you would prefer to receive an amount of money for yourself or an amount of money for the person listed. Please circle A or B for each line.

Each of the next six pages summarized the above instructions and then presented a list of questions as follows, with a different $N$-value on each page:

Now imagine the following choices between an amount of money for you and an amount for you and for your friend. Circle A or B to indicate which you would choose in EACH line.

120,000 yen for you alone

60,000 yen for you and 60,000 yen for your friend

110,000 yen for you alone

60,000 yen for you and 60,000 yen for your friend

..(continuing down to).

(A) 60,000 yen for you alone

(B) 60,000 yen for you and 60,000 yen for your friend 
The A-rows listed 9 amounts decreasing by 10,000 yen on each line, from 120,000 to 60,000 yen. Row-B had a different degree of separation on each page. The degrees of separation were from one to six. On each line, the participants were asked to choose between an amount of money just for themselves and 60,000 yen each for themselves and for the person. The degrees of separation were manipulated by adding "of friend" to the sentence in line B. For example, the second order friend was described as the "friend of your friend," and the third order friend as the "friend of the friend of your friend." All participants finished their questionnaire within fifteen minutes.

\section{Results and Discussion}

The crossover point was estimated as the average of the last selfish (row-A) choices and the first generous (row-B) choices, in the same way as in Jones and Rachlin (2006) or Rachlin and Jones (2008). For example, if a participant preferred 90,000 yen for herself to having 60,000 yen and giving 60,000 yen to the Nth degree of separation friend, but preferred to have 60,000 yen and give 60,000 yen to the Nth friend over having 80,000 yen for herself, then the crossover point was taken as being 85,000 yen for that participant at that $N$-value. Some participants chose the generous option even when the alternative was 120,000 yen for themselves. In these cases, a crossover point of 125,000 was assumed. In contrast, many participants chose the selfish option even when the choice was between 60,000 yen for themselves and 60,000 yen for themselves in addition to 60,000 yen for their friends. In these cases, the crossover point was assumed to be 0 .

Figure 1 shows the mean allocation of money to friends who have $n$ degrees of separation. We fitted both the hyperbolic and exponential functions to this data and compared their performances. The solid line is the best fitting hyperbolic discount function and the dashed line is the best fitting exponential discount function. The fit is remarkably good $\left(R^{2}=0.995\right)$ when compared with the fit of intertemporal choice (e.g., Rachlin \& Raineri, 1992) or of social discounting (Jones \& Rachlin, 2006).

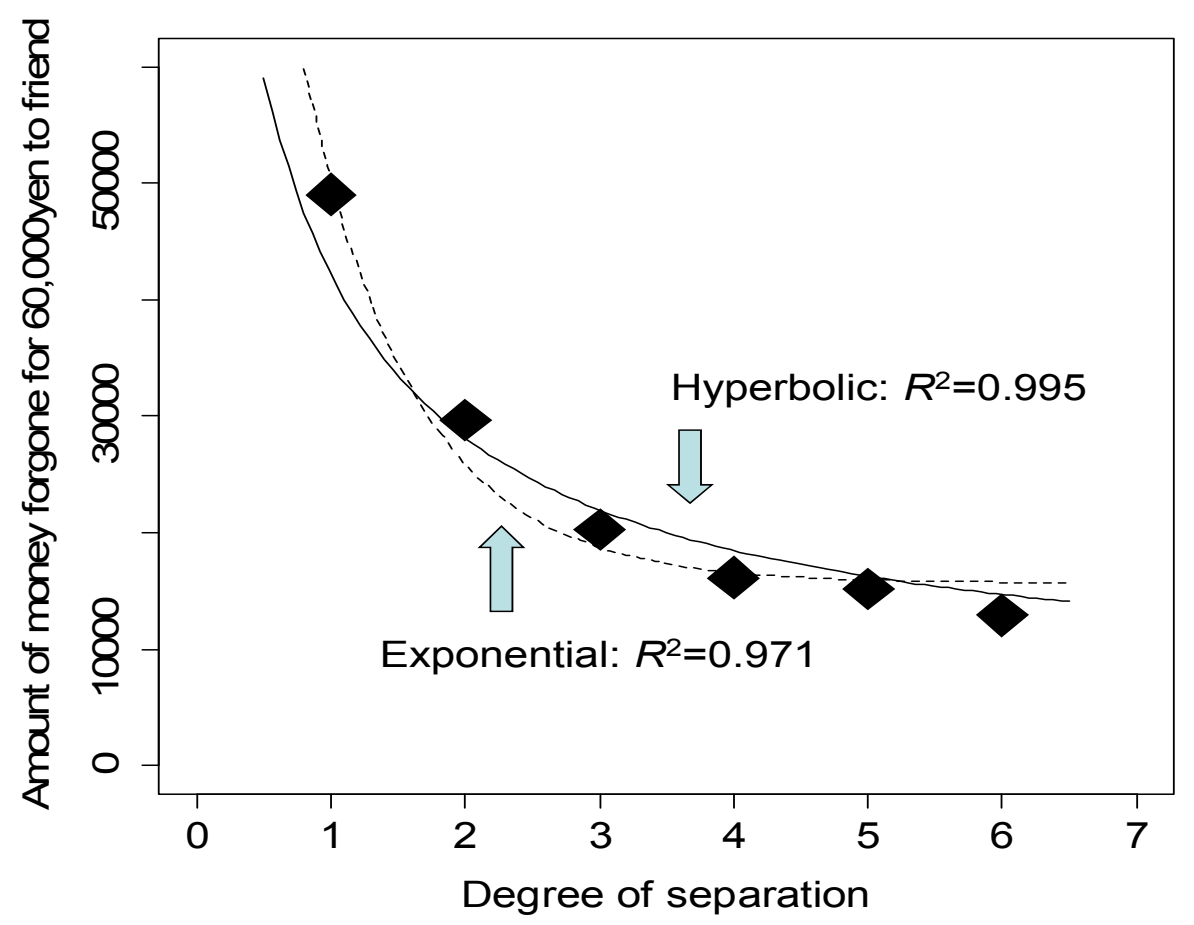

Figure 1. Hyperbolic and exponential discount functions under the $n$th degree of separation in Study 1. 
For comparison, the best fitting exponential discount function is also shown in Figure 1, as the dashed line. Although the fit with exponential discounting is high $\left(R^{2}=0.971\right)$, the percentage of variance accounted for by the exponential discount function is less than that of the hyperbolic function. The difference found between the fit of the hyperbolic and the exponential discounting is almost the same as that in Jones and Rachlin (2006). In sum, these results show that the hyperbolic function provides a better fit to the data than the exponential discount function. Thus, we can conclude that people's discounting in terms of degrees of separation is similar to that of social ranking and intertemporal choice.

\section{Study 2}

Study 2 was designed to investigate a relationship between $\mathrm{n}$ degrees of separation and social rankings. Although the two are similar as they both represent some kind of social distance, they do differ as while the latter distance can only represent the remoteness of in-group members, the former includes that of both in-group and out-group people. Thus, although Study 1 demonstrated hyperbolic discounting occurs in $n$ degrees of separation, the way in which social ranking and degrees of separation are similar types of social distance still remains unclear. Therefore, Study 2 required participants to indicate their preference between receiving money for themselves and sharing money with their friends, under both social ranking and degrees of separation types of distance.

\section{Procedure}

Two hundred and fourteen participants answered social discounting tasks using both social ranking and degree of separation conditions. As tasks to explore preferences with social distance as degrees of separation, we employed the same tasks as those in Study 1. For the social ranking tasks, we employed a procedure almost the same as that of Jones and Rachlin (2006). Precisely, the participants were provided with instructions that had been translated into Japanese from the original ones used by Jones and Rachlin (2006). Then participants were required to make choices between receiving an amount of money for themselves or receiving an amount of money for themselves and their friends, using the following response form:

Now imagine the following choices between an amount of money for you and an amount for you and for your friend. Circle A or B to indicate which you would choose in EACH line.

(A) 120,000 yen for you alone

(B) 60,000 yen for you and 60,000 yen for your \#_person

(A) 110,000 yen for you alone

(B) 60,000 yen for you and 60,000 yen for your \#_person ..(continuing down to).

(A) 60,000 yen for you alone

(B) 60,000 yen for you and 60,000 yen for your \#_person

The blanks shown above was replaced by a number $(N=1,2,5,10,20,50$, or 100), with a different number used on each of the seven question pages.

Participants answered both the social ranking and degree of separation tasks in random order. All participants completed all the tasks within 20 minutes.

\section{Results and Discussion}

Figure 2 shows the results of both the social ranking and the degree of separation tasks. We found that the 
hyperbolic function fitted the data better than the exponential function in both cases. The differences between the hyperbolic and exponential discounting in Study 2 are more remarkable than those in Study 1, or in Jones and Rachlin (2006); whereas the $R^{2} \mathrm{~S}$ of the exponential functions are 0.873 and 0.850 , those of the hyperbolic functions are greater than 0.90 . Additionally, the data points systematically deviate from the exponential functions. Thus, we can conclude that Study 2 replicated the results of Study 1 and of Jones and Rachlin (2006).
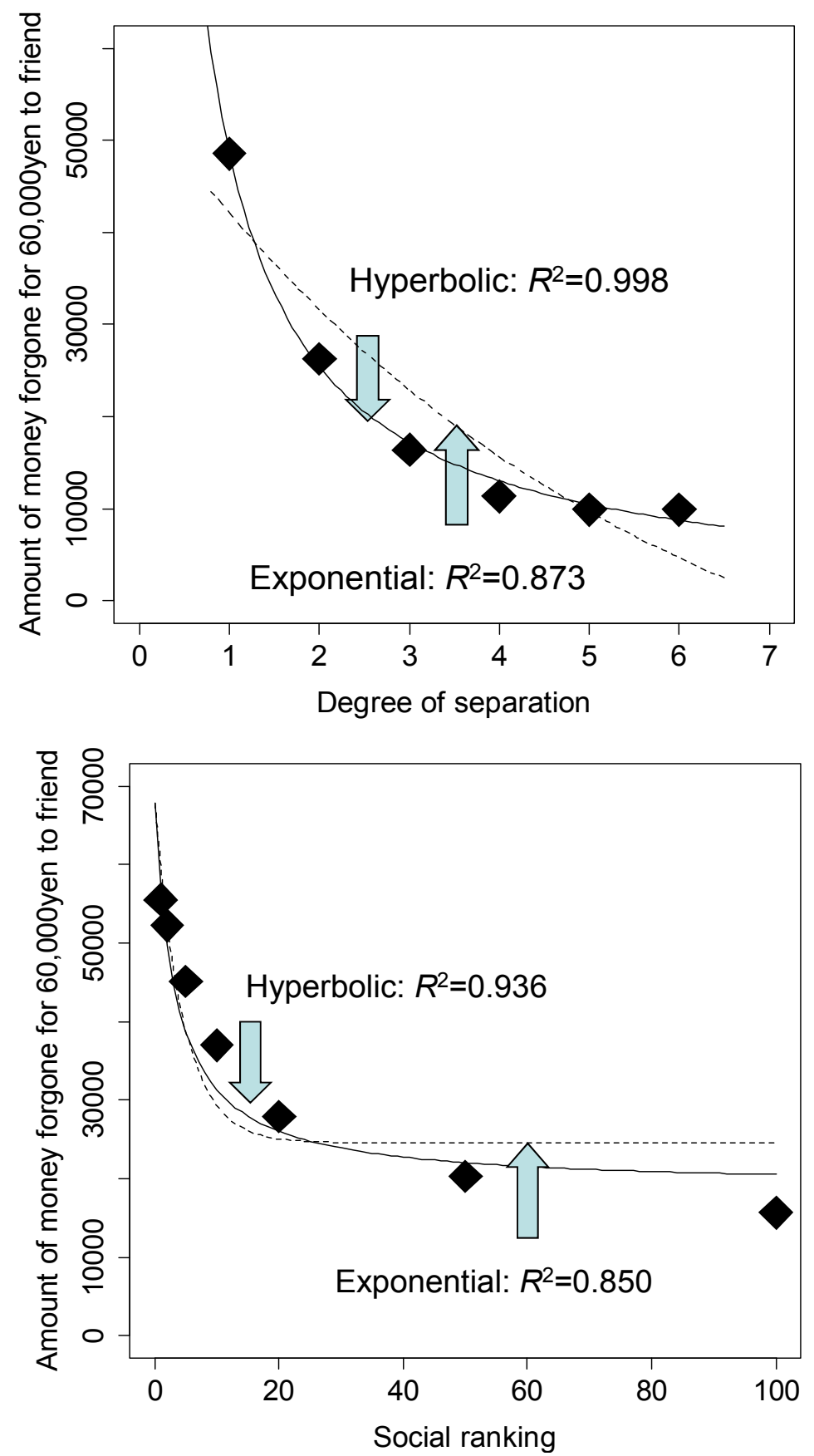

Figure 2. Hyperbolic and exponential functions with data from the $\mathrm{n}$ degrees of separation and social ranking tasks in Study 2: The upper graph shows results in the $\mathrm{n}$ degrees of separation case, and the lower shows those in the social ranking case. 
To explore the relationship between social ranking and degrees of separation in depth, we performed the following two additional analyses. First, we estimated the $k$ parameters of hyperbolic functions for the social ranking and degree of separation tasks. There was no relationship $(r=-0.03, p>0.01$ : see Figure 3$)$ between $k$ parameters in the two tasks (see Figure 3), indicating that the steepness of the functions in the social ranking and degrees of separation cases are somewhat independent from each other.

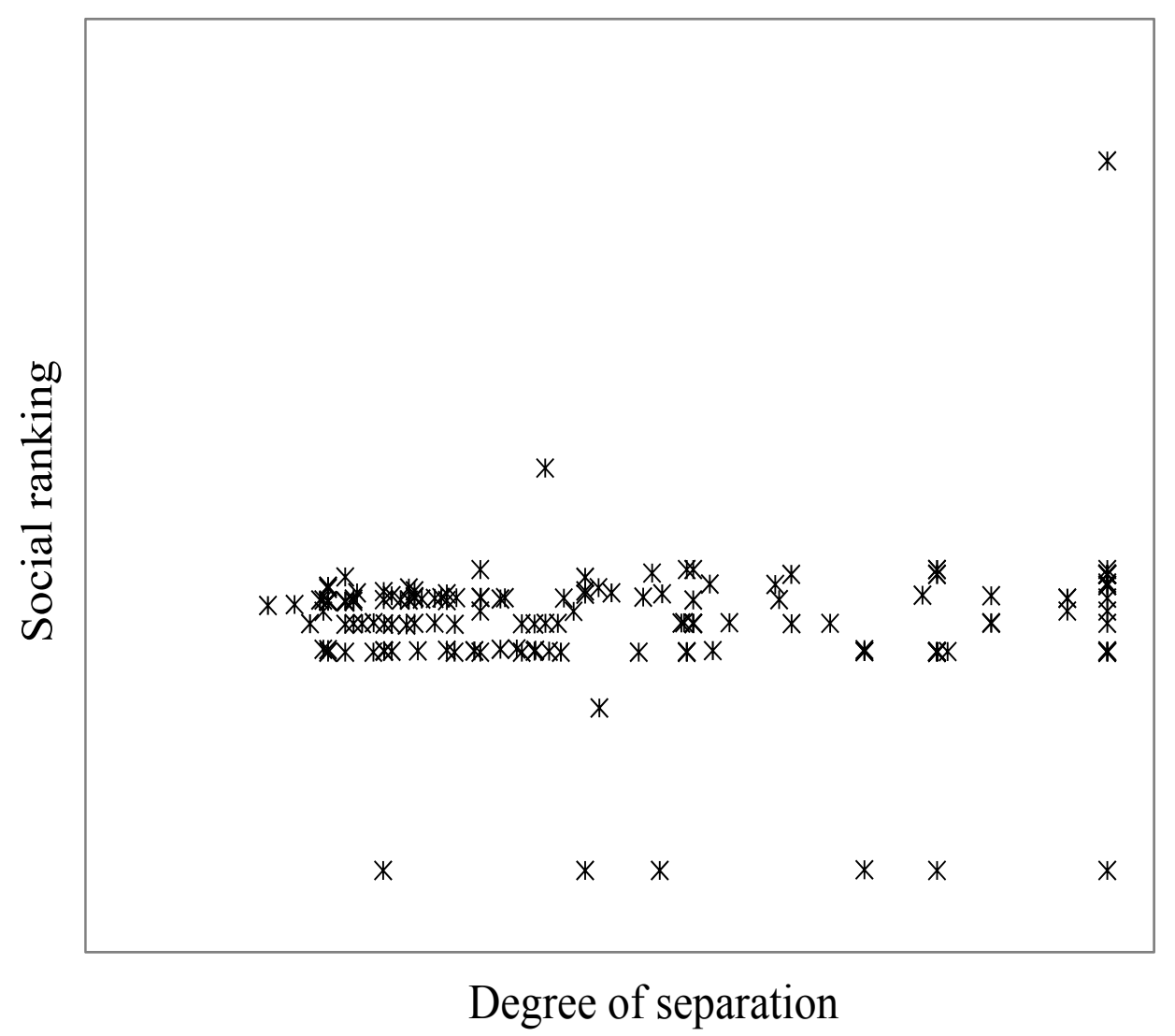

Figure 3. Scatterplot of $k$ parameter.

Second, we also performed a factor analysis of the crossover points with promax rotation using the maximum likelihood method. The eigenvalues for the first three factors were 7.04, 2.54, and 1.35, respectively. Mainly due to the eigenvalue results, we adopted a three-factor solution pattern for the discounting tasks shown in Table 1, assuming the following interpretations of the meaning of the three factors.

Factor 1 leads mainly to allocations for high ranking friends and first degree friends. To define, these friends can be interpreted as close friends, so we named this factor "close friends". Factor 2 can be considered as the "unknown others" factor, because it strongly influences the allocation to friends who have more than one degree of separation. Logically, one cannot directly know friends who are of more than the second order, and specifically, friends who have more than two degrees of separation are actually unknown because they are not even the friend of a first order friend. Factor 3 mainly impacts friends with rankings higher than 10th, and in particular those who are ranked at 20th place or higher. These friends are considered as not being so close. Thus, we named this factor as the "acquaintances" factor. 
Table 1

Results of Factor Analysis

\begin{tabular}{llll}
\hline & Factor 1 & Factor 2 & Factor 3 \\
\hline Degree of separation & & & -0.07 \\
1st & 0.78 & 0.12 & 0.10 \\
2nd & 0.35 & 0.43 & 0.07 \\
3rd & 0.11 & 0.75 & 0.06 \\
4th & -0.03 & 0.94 & -0.02 \\
5th & -0.05 & 0.99 & -0.03 \\
6th & -0.06 & 0.99 & -0.10 \\
\hline Social ranking & & & -0.07 \\
1st & 1.00 & -0.03 & 0.23 \\
2nd & 1.01 & -0.03 & 0.53 \\
5th & 0.79 & -0.06 & 0.79 \\
10th & 0.50 & -0.08 & 0.97 \\
20th & 0.26 & -0.11 & 0.83 \\
50th & -0.09 & 0.05 & \\
100th & -0.16 & 0.18 & \\
\hline Correlations & & & 1.00 \\
Factor 1 & 1.00 & 1.00 & \\
Factor 2 & 0.36 & 0.49 & \\
Factor 3 & 0.53 & &
\end{tabular}

The above results suggest that there are several dimensions to social discounting. Specifically, it is interesting that people have two dimensions of social distance with others who are not so close to them. To examine this indication more precisely, we also performed structural equation modeling and compared models of one, two, and three factors (see Figure 4).

The one factor model represents a hypothesis that both social rankings and degrees of separation can be summarized by one dimension, that people's dimension of social discounting is unitary. In contrast, the two and three factor models assume that social discounting can be decomposed into several dimensions. The two-factor model entails that social discounting occurs separately in the social ranking and the $n$ degrees of separation tasks. In other words, this model assumes that participants construct a dimension of social discounting in accordance with experimental tasks. The three-factor model expresses an implication, based on the results of the factor analysis, that the two types of social distance considered in this study can be decomposed into three factors: one influences both social ranking and degrees of separation, and the other two factors affect these two dimensions, respectively.

The results of the structural equation modeling, shown in Table 2, clearly support the three factor model, as all of the fit indices indicate it is superior to the other two models. Thus, we can conclude that although social discounting in social rankings and degrees of separation share the same components, they can be decomposed into several dimensions. That is, while these two types of social distance are similar in how they reflect allocations made to closer friends, they differ in representing the allocations made to others who are not so close. 


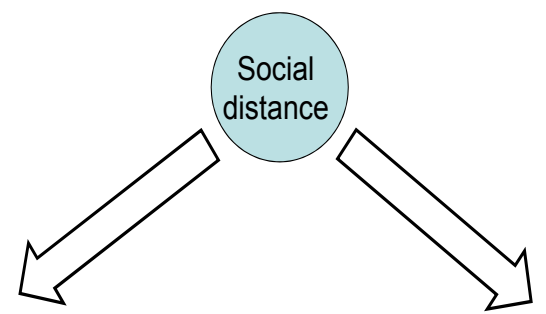

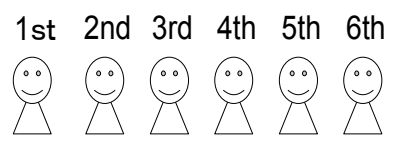

$\mathrm{N}$ degree of separation

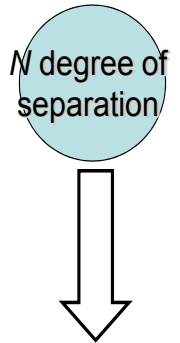

1st 2nd 3rd 4th 5th 6th

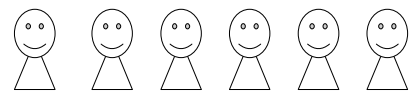

$\mathrm{N}$ degree of separation

1st 2nd 5th 10th 20th 50th 100th

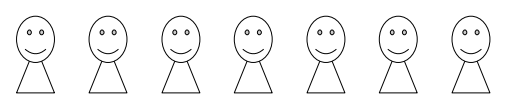

Social ranking

(a)

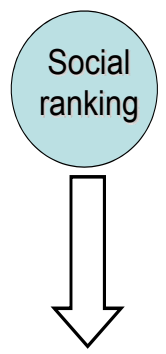

1st 2nd 5th 10th 20th 50th 100th

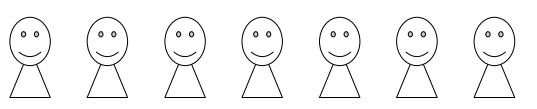

Social ranking

(b)

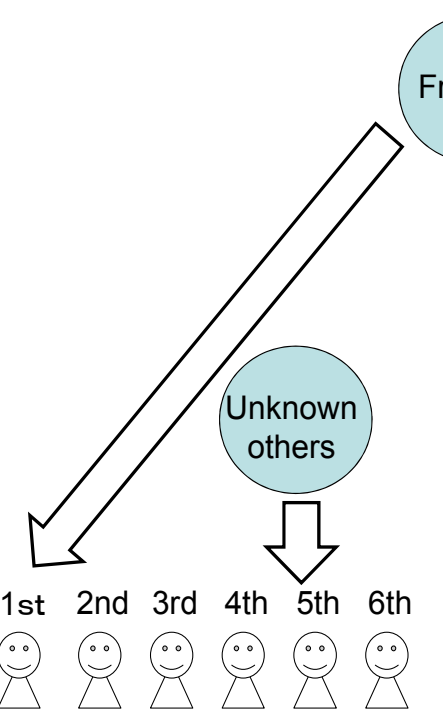

$\mathrm{N}$ degree of separation

ds Friends
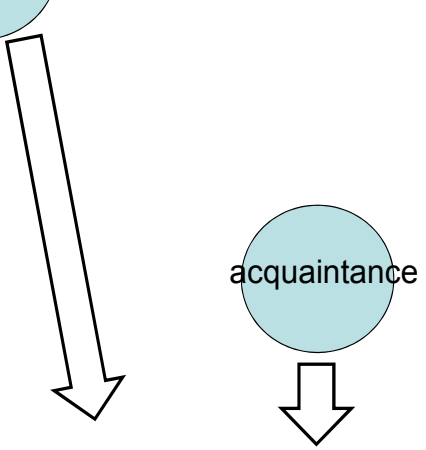

1st 2nd 5th 10th 20th 50th 100th

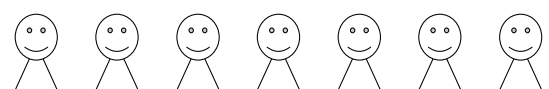

Social ranking

(c)

Figure 4. Three structural equation models: (a) a one-factor model that indicates both the social ranking and $\mathrm{n}$ degrees of separation can be summarized by one dimension; (b) a two factor model that implies participants construct dimensions of social discounting corresponding to the experimental tasks; and (c) a three-factor model that reflects the implication of the factor analysis that social rankings and degrees of separation share one common factor ("friend"), but are also individually affected by "acquaintances" and "unknown others" factors, respectively. 
Table 2

Results of Structural Equation Modeling

\begin{tabular}{lllll}
\hline & AIC & BIC & GFI & CFI \\
\hline Model 1 & 311.98 & 497.11 & 0.88 & 0.96 \\
Model 2 & 342.47 & 534.33 & 0.90 & 0.95 \\
Model 3 & 186.37 & 331.11 & 0.94 & 0.99 \\
\hline
\end{tabular}

\section{General Discussion}

The results of the two studies can be summarized as follows. First, we found that there is hyperbolic social discounting with the $n$ degrees of separation type of social distance. Recently, many researchers have paid attention to the way in which the structure of our social networks affects human life (e.g., Christarski \& Fowler, 2009). Most of these studies investigate how people's behavior affects others through links between persons. However, previous studies have not considered the way in which people consider others in their social network. In this vein, this may be the first study that concerns how people consider others who have $n$ degrees of separation. Furthermore, as far as we know, this is the first study that shows hyperbolic discounting occurring with social distance other than in closeness rankings. Hyperbolic discounting is found not only in the domain of social discounting but also in probability or intertemporal discounting (Jones \& Rachlin, 2009). Thus, this study applied hyperbolic discounting to another type of social distance and has demonstrated with evidence the hyperbolic function's ability to explain discounting under various types of psychological distance.

Second, from the results of estimating the parameters of hyperbolic discounting and the structural equation modeling, we can conclude that people have two dimensions to their conceptualization of others who are not so close to them: One applies to others who are known but are not so close, and the other applies to those who are unknown. Intuitively, these appear to be quite natural results. However, these results are interesting because hyperbolic discounting occurs under both dimensions, despite them being independent of each other. Additionally, this finding is also of interest because it appears to contradict Simon's (1995) suggestion that social discounting is one-dimensional.

The results of this study may support construal level theory (Trope \& Lieberman, 2010). This theory assumes that people's judgments of various types of psychological distance can be decomposed into two levels of construal: higher and lower levels. The theory claims that high-level construals are relatively abstract, coherent, and super-ordinate mental representations as compared with low-level construals. It also argues that people use increasingly higher levels of construal to represent an object as the psychological distance from the object increases. The results of our factor analysis and structural equation modeling consistently show that both the social ranking and $n$ degrees of separation can be decomposed into two factors. One factor reflects allocations made to psychologically close friends, and the other reflects those to psychologically remote friends. This factor structure appears to match the structure entailed by construal level theory.

In addition, what is more interesting in the results of the factor analysis is that the factor reflecting allocations to close friends is related to both the social ranking and $n$ degrees of separation types of distance. As stated in the introduction, social rankings and degrees of separation are somewhat different dimensions of social distance: while the former represents distances between known others, the latter contains those between unknown others. In this vein, the results of the factor analysis suggest that the contents of the "friends" factor are richer than those of the other two factors, because this factor influences the two different types of social 
dimension. If we interpret the "friends" factor as a lower level construal that is psychologically closer, this indication corresponds to a proposition of construal level theory that lower level construals have more complex representations than higher level construals (Trope \& Lieberman, 2010).

One may suspect that current results solely reflects participants' perception for "number" rather than that for social distance. That is, social distances employed in this study such as social ranking or degree of separation is somewhat abstract for participants, so there is a possibility that participants could not grasp precise images for these distances and thus might respond depending on their perception for magnitude of numbers used in this study.

This question is important and should be explored in the future research because it concerns meaning of social distance: it is sure that social distance is a very abstract concept and at least ordinary people rarely rank their friends or consider how remote friend of their friend is more them. In this vein, a question that how participants distinctively understand meanings of rank or degree of separation is critical for experimental aspect of studies on social discounting.

However, as far as this study concerns, the results of factor analysis can eliminate the above interpretation. The factor pattern shown in Table 1 indicate that there is a prominent distinction among the three factors, suggesting that participants surely had precise distinction among "close friend", "acquaintances", and "unknown others". This distinction matches experimental manipulation of this study, supporting a possibility that participants' judgments reflect their perception for various dimension of social distance.

Of course, the following possibility still remains: it is sure that participants' judgments are based on their perception for social distances, but good fit of the hyperbolic function in social discounting is due to their perception for number. This demarcation should be investigated not only in the domain of social discounting but also other domains of discounting including intertemporal discounting (e.g., Lowenstein \& Thaler, 1989) or probability discounting (for a review, see Vulkan, 2000). Thus, one more important issue for future research regarding social discounting in cases of $n$ degrees of separation may be its relationship to probability or intertemporal discounting. Jones and Rachlin (2009) reported that the steepness of discounting, represented by the $k$ parameter, was correlated for probability, intertemporal, and social ranking discounting across participants. The results of Study 2 show that the $k$ parameter did not correlate between the social ranking and $n$ degrees of separation experiments, suggesting that the latter dimension may be unique and different from the other three dimensions. If so, what is the meaning of social discounting? Why does the $n$ degrees of separation differ from delay, probability, and social ranking in its discounting? Pursuing this question may be fruitful in exploring how "social" is represented in the human mind.

\section{References}

Barabási, A. L. (2002). Linked: The new science of networks. Perseus Books Group.

Christakis, N. A., \& Fowler, J. (2009). Connected: The surprising power of our social networks and how they shape our lives. Little, Brown and Company.

Green, L., \& Myerson, J. (2002). A discounting framework for choice with delayed and probabilistic reward. Psychological Bulletin, 130, 769-792.

Jones, B., \& Rachlin, H. (2006). Social discounting. Psychological Science, 17, 283-286.

Jones, B., \& Rachlin, H. (2009). Delay, probability and social discounting in a public goods game. Journal of Experimental Analysis of Behavior, 91, 61-73.

Lowenstein, G., \& Thaler, R. (1989). Intertemporal choice. Journal of Economic Perspective, 3, 181-193. 
Mazur, J. E. (1987). An adjusting procedure for studying delayed reinforcement. In M. L. Commons, J. E. Mazur, J. A. Nevin, \& H. Rachlin (Eds.), Quantitative analysis of behavior Vol. 5: The effects of delay and of intervening events on reinforcement value (pp. 55-73). Mahwah, N.J.: Erlbaum.

Milgram, S. (1967). The small-world problem. Psychology Today, 1, 60-67.

Rachlin, H., \& Jones, B. (2008). Social discounting and delay discounting. Journal of Behavioral Decision making, 21, 29-43.

Rachlin, H., \& Raineri, A. (1992). Irrationality, impulsiveness, and selfishness as discount reversal effects. In G. F. Loewenstein, \& J. Elster (Eds.), Choice over time (pp. 93-118). New York: Russell Sage Foundation.

Simon, J. L. (1995). Interpersonal allocation continuous with intertemporal allocation: binding commitments, pledges, and bequests. Rationality and Society, 7, 367-392.

Trope, Y., \& Lieberman, N. (2010). Construal-level theory of psychological distance. Psychological Review, 117, 440-463.

Vulkan, N. (2000). An economist's perspective on probability-matching. Journal of Economic Surveys, 14, 101-118. 